\title{
O fenômeno El Niño Oscilação Sul e a variabilidade interanual da evaporação do tanque Classe A e da umidade relativa do ar em Santa Maria, RS
}

\author{
El Niño Southern Oscilation and the interannual variability of Pan evaporation and air relative \\ humidity in Santa Maria, RS, Brazil
}

\author{
Nereu Augusto Streck ${ }^{\mathrm{I}^{*}}$ Hamilton Telles Rosa ${ }^{\mathrm{II}}$ Lidiane Cristine Walter $^{\mathrm{II}}$ Leosane Cristina Bosco ${ }^{\mathrm{III}}$ \\ Isabel Lago ${ }^{\text {III }}$ Arno Bernardo Heldwein ${ }^{I}$
}

\begin{abstract}
O objetivo deste trabalho foi associar a variabilidade interanual da evaporação do tanque Classe A e da umidade relativa do ar com o fenômeno El Niño Oscilação Sul (ENOS) em Santa Maria, RS. Foram utilizados os dados diários de evaporação do tanque Classe A (ECA, $\left.\mathrm{mm} \mathrm{dia}^{-1}\right) e$ umidade relativa média diária do ar (UR, \%) medidos em Santa Maria, RS. A ECA foi medida de 1973 a 2006 e a UR de 1969 a 2006. Os anos foram classificados em El Niño (EN), La Niña (LN) e Neutros (N), considerando o período de 01/07 de um ano até 30/06 do ano seguinte. Os resultados mostraram que a ECA é menor nos anos de EN e maior nos anos de LN. Já a UR foi maior em anos de EN e menor em anos de LN. O efeito do fenômeno ENOS sobre a ECA é maior nos meses de novembro, dezembro, janeiro e maio, enquanto que sobre a UR os meses de maior influência do ENOS são outubro, novembro, dezembro e maio.
\end{abstract}

Palavras-chave: La Niña, vapor d’água, variabilidade climática, risco climático, agricultura.

\section{ABSTRACT}

The objective of this study was to associate the interannual variability of Pan evaporation and air relative humidity with the El Niño Southern Oscilation (ENSO) phenomenon in Santa Maria, RS, Brazil. Daily data records of Pan evaporation (PAN, $\mathrm{mm} \mathrm{day}^{-1}$ ) and mean daily relative humidity $(\mathrm{RH}, \%)$ measured in Santa Maria, RS, were used. PAN was measured from 1973 to 2006 and $R H$ was measured from 1969 to 2006. Years were grouped into El Niño (EN) years, La Niña (LN) years, and Neutral (N) years, from July $1^{\text {st }}$ of the year to June $30^{\text {th }}$ of the following year. Results showed that PAN is lower in EN years and greater in $L N$ years. On the other hand, $R H$ was greater in EN years and lower in $L N$ years.

\begin{abstract}
The effect of ENSO on PAN is greater in November, December, January, and May, whereas RH is affected by ENSO in October, November, December and May.
\end{abstract}

Key words: La Niña, water vapor, climate variability, climate risk, agriculture.

O fenômeno El Niño Oscilação Sul (ENOS) é um fenômeno de grande escala que acontece na região do Oceano Pacífico equatorial, constituído de dois componentes, um oceânico e outro atmosférico, e de duas fases, a fase quente (El Niño) e a fase fria ( $\mathrm{La}$ Niña) (GLANTZ, 2001; BERLATO \& FONTANA, 2003). No Brasil, o sinal do ENOS se dá, principalmente, por anomalias climáticas de precipitação pluviométrica das regiões Sul e Nordeste (GRIMM et al., 1996a,b; OLIVEIRA \& SATYAMURTY, 1998; MARENGO \& OLIVEIRA, 1998; DIAZ et al., 1998; BERLATO \& FONTANA, 2003). Entre os elementos meteorológicos que ainda não foram associados com o fenômeno ENOS no Rio Grande do Sul, estão a evaporação do tanque Classe A (ECA) e a umidade relativa do ar (UR). AECA representa a lâmina de água transferida de reservatórios de água livre para a atmosfera e por meio desta podese estimar a necessidade hídrica, a lâmina e o turno de rega na irrigação das lavouras (MATZENAUER et al., 1998; CAMPELO JÚNIOR \& CURI, 2001; CARLESSO et al., 2001). A UR é um dos principais elementos meteorológicos que influenciam o estabelecimento e o

IDepartamento de Fitotecnia, Centro de Ciências Rurais (CCR), Universidade Federal de Santa Maria (UFSM). Avenida Roraima, 1000, 97105-900, Santa Maria, RS, Brasil. E-mail: nstreck1@smail.ufsm.br. *Autor para correspondência.

${ }^{\text {IIC }}$ urso de Agronomia, CCR, UFSM, Santa Maria, RS, Brasil.

IIIPrograma de Pós-graduação em Engenharia Agrícola, CCR, UFSM, Santa Maria, RS, Brasil. 
desenvolvimento de epifitias em plantas (HELDWEIN, 1997; SENTELHAS et al., 2004), o ataque de insetos como ácaros (BOUDREAUX, 1958; ENGLISH-LOEB, 1990), a secagem ou desidratação da planta para fenação (VILELA, 1994) e o risco de incêndios florestais e de campos (SOARES, 1984). O objetivo deste trabalho foi associar a variabilidade interanual da evaporação do tanque Classe A e da umidade relativa do ar com o fenômeno ENOS em Santa Maria, RS.

Foram usados a evaporação média diária medida no tanque Classe A(ECA, mm dia ${ }^{-1}$ ) e a umidade relativa média diária do ar (UR,\%) medidos na Estação Climatológica Principal do $8^{\circ}$ Distrito de Meteorologia, localizada no Departamento de Fitotecnia da Universidade Federal de Santa Maria (latitude: 2943'S, longitude: 53\%48'W e altitude: 95m). Usaram-se os dados de ECA de 01/07/1973 a 30/06/2006, totalizando 33 anos, e UR do período de 01/07/1969 a 30/06/2006, totalizando 37 anos. A UR foi calculada a partir de três leituras diárias (9 horas, 15 horas e 21 horas): $\mathrm{UR}=\left(\mathrm{UR}_{\mathrm{9h}}+\right.$ $\left.\mathrm{UR}_{15 \mathrm{~h}}+2 . \mathrm{UR}_{21 \mathrm{~h}}\right) / 4$. Nos dias de chuva em que houve transbordamento de água do tanque Classe A, considerou-se os dados de ECA como dados perdidos.

Classificaram-se os anos em anos de El Nino (EN), La Niña (LN) e Neutros (N), segundo BERLATO et al. (2005) e NOAA (2007), considerando-se o período de 01/07 do ano até 30/06 do ano seguinte, já que o fenômeno ENOS geralmente inicia no segundo semestre de um ano e acaba no primeiro semestre do ano seguinte (BERLATO \& FONTANA, 2003; BERLATO et al., 2005). Os anos de El Niño foram 1969-1970, 1972-1973, 19761977, 1982-1983, 1986-1987, 1987-1988, 1991-1992, 19921993, 1993-1994, 1994-1995, 1997-1998, 2002-2003 e 20042005; os anos de La Niña foram 1970-1971, 1971-1972, 1973-1974, 1974-1975, 1975-1976, 1984-1985, 1988-1989,
1995-1996, 1998-1999 e 1999-2000; os anos Neutros foram 1977-1978, 1978-1979, 1979-1980, 1980-1981, 19811982, 1983-1984, 1985-1986, 1989-1990, 1990-1991, 19961997, 2000-2001, 2001-2002, 2003-2004 e 2005-2006. Os resultados foram analisados por análise de probabilidade empírica (diagrama de caixa) em nível mensal e anual (BERLATO \& FONTANA, 2003).

Os diagramas de caixa anual para ECA e UR são apresentados na figura 1, onde lê-se, por exemplo, que para a probabilidade de $90 \%$, em $90 \%$ dos casos a ECA e a UR estão abaixo dos valores correspondentes aos valores indicados pela extremidade superior da barra. O valor de ECA na probabilidade de $90 \%$ foi menor em anos de EN, maior em anos de LN e intermediário em anos N (Figura 1A) e o valor da UR nesta probabilidade foi maior em anos de EN, menor em anos de LN e intermediário em anos N (Figura 1B). Os menores valores de ECA e maiores valores de UR em anos de EN estão associados a condições meteorológicas de maior nebulosidade e maior número de dias com chuva nestes anos (CARMONA \& BERLATO, 2002; FONTANA \& ALMEIDA, 2002). Já os maiores valores de ECA e menores valores de UR em anos de LN estão associados a uma maior insolação relativa e menor precipitação pluvial nestes anos (FONTANA \& BERLATO, 1997; CARMONA \& BERLATO, 2002). Os diagramas de caixa mensais da ECA e da UR (dados não mostrados) indicaram que os meses de maior influência do fenômeno ENOS sobre a ECA e UR foram novembro, dezembro, janeiro e maio para a ECA e outubro, novembro, dezembro e maio para a UR. Estes meses coincidem com o primeiro e o segundo trimestre de maior influência do fenômeno ENOS sobre a precipitação pluvial do Rio Grande Sul, que são outubro a dezembro e abril a junho, respectivamente (FONTANA \& BERLATO, 1997).
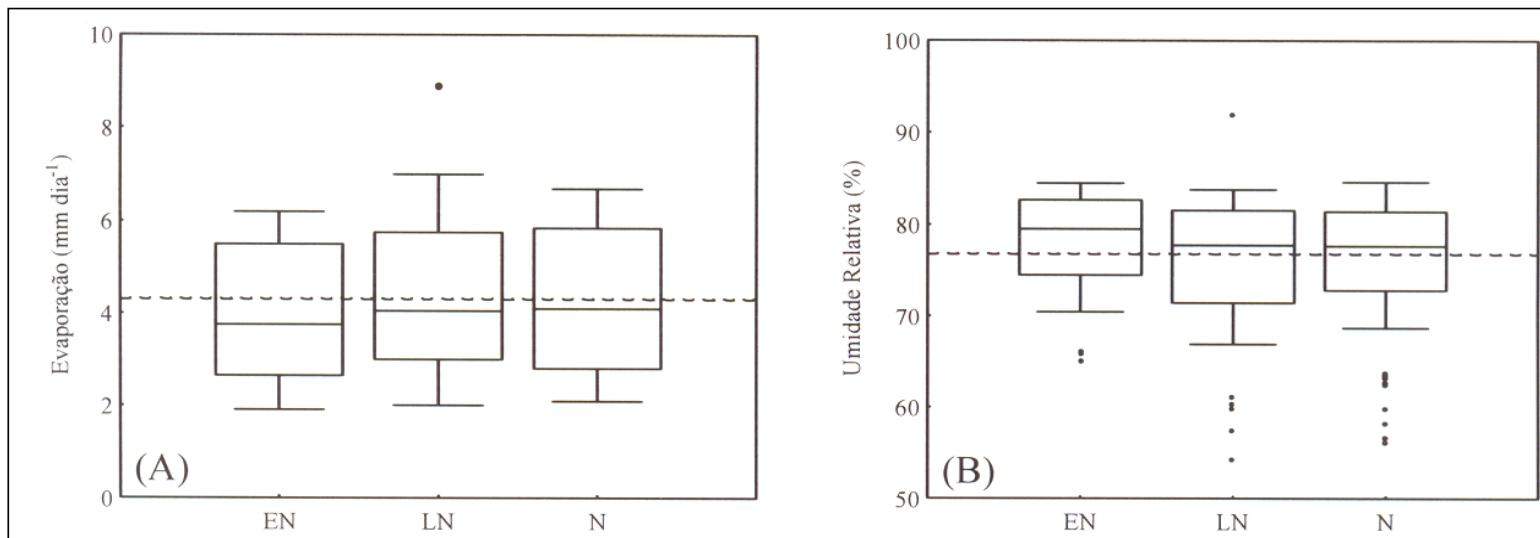

Figura 1 - Distribuição da evaporação média mensal medida no tanque Classe A no período de 1973 a 2006 (A) e da umidade relativa do ar média mensal no período de 1969 a 2006 (B) associada ao anos de El Niño (EN), La Niña (LN) e Neutros (N), para Santa Maria, RS. A linha cheia horizontal, no interior das caixas, representa o percentil de 50 (mediana); a linha tracejada representa a média dos anos neutros; o final das caixas representa os percentis de 25 e 75 ; as barras representam os percentis de 10 e 90 ; os pontos, quando presentes, representam os valores extremos. 
Os resultados deste estudo podem auxiliar no planejamento das atividades agrícolas visando reduzir os riscos do agronegócio. Por exemplo, na previsão de um evento de El Niño, são esperados menores valores de evaporação do tanque classe A e maiores valores de umidade relativa do ar, o que deve levar a um menor uso de água pelas culturas de verão (MATZNAUER et al., 1998; CAMPELO JUNIOR \& CURI, 2001; CARLESSO et al., 2001), condições mais favoráveis ao aparecimento de doenças nas lavouras (HELDWEIN, 1997; SENTELHAS et al., 2004) e dificuldade na secagem de material vegetal para fenação (VILELA, 1994). Ao contrário, se a previsão é de La Niña, esperam-se maiores valores de evaporação do tanque classe A e menores valores de umidade relativa do ar e, neste caso o agricultor pode se preparar para um aumento na necessidade de água pelas culturas (MATZNAUER et al., 1998; CAMPELO JUNIOR \& CURI, 2001; CARLESSO et al., 2001), o que implica em um ajuste da área plantada ao tamanho dos reservatórios de água na propriedade, bem como condições mais favoráveis ao aparecimento de insetospraga como ácaros, os quais são favorecidos por períodos secos e baixa umidade relativa do ar (50-65\%) (BOUDREAUX, 1958; ENGLISH-LOEB, 1990), e maior risco de incêndios florestais e em campos (SOARES, 1984).

\section{AGRADECIMENTOS}

Ao Conselho Nacional de Desenvolvimento Científico e Tecnológico (CNPq), à Fundação de Amparo à Pesquisa do Estado do Rio Grande do Sul (FAPERGS) e à Coordenação de Aperfeiçoamento de Pessoal de Nível Superior (CAPES), pelas bolsas concedidas.

\section{REFERÊNCIAS}

BERLATO, M.A.; FONTANA, D.C. El Niño e La Niña: impactos no clima, na vegetação e na agricultura do Rio Grande do Sul; aplicações de previsões climáticas na agricultura. Porto Alegre: UFRGS, 2003. 110p.

BERLATO, M.A. et al. Associação entre El Niño oscilação Sul e a produtividade de milho no Estado do Rio Grande do Sul. Pesquisa Agropecuária Brasileira, v.40, p.423-432, 2005.

BOUDREAUX, H.B. The effect of relarive humidity on egglaying, hatching and surviral in various spider mites. Jounal of Insect Physiology, v.2, p.191-218, 1958.

CARLESSO, R. et al. Irrigação por aspersão no Rio Grande do Sul. Santa Maria: UFSM, 2001. 165p.

CAMPELO JÚNIOR, J.H.; CURI, S. Necessidades hídricas da cultura do feijoeiro (Phaseolus vulgaris L.) na baixada Cuiabana.
Revista Brasileira de Agrometeorologia, v.9, n.1, p.5965, 2001.

CARMONA, L.C.; BERLATO, M.A. El Niño e La Niña e o rendimento do arroz irrigado no Estado do Rio Grande do Sul. Revista Brasileira de Agrometeorologia, v.10, n.1, p.147152, 2002.

DIAZ, A.F. et al. Relationships between precipitation anomalies in Uruguay and southern Brazil and sea surface temperature in the Pacific and Atlantic Oceans. Journal of Climate, v.11, p.251-271, 1998.

ENGLISH-LOEB, G.M. Plant drought stress and outbreaks of spider mites: a field test. Ecology, v.71, n.4, p.1401-1411, 1990 .

FONTANA, D.C.; ALMEIDA, T.S. Climatologia do número de dias com precipitação pluvial no Estado do Rio Grande do Sul. Revista Brasileira de Agrometeorologia, v.10, n.1, p.135145, 2002.

FONTANA, D.C.; BERLATO, M.A. Influência do El Niño Oscilação Sul sobre a precipitação do Estado do Rio Grande do Sul. Revista Brasileira de Agrometeorologia, v.5, n.1, p.127-132, 1997.

GLANDZ, M.H. Currents of change: impacts of El Niño and La Niña on climate and society. 2.ed. Cambridge: University, 2001. 252p.

GRIMM, A.M. et al. Anomalias de precipitação no sul do Brasil em eventos El Niño. In: CONGRESSO BRASILEIRO DE METEOROLOGIA, 9., 1996. Campos do Jordão, SP. Anais... Campos do Jordão: Sociedade Brasileira de Meteorologia, 1996a. 2v. 1250p. p.1098-1102.

GRIMM, A.M. et al. Anomalias de precipitação no sul do Brasil em eventos La Niña. In: CONGRESSO BRASILEIRO DE METEOROlogiA, 9., 1996. Campos do Jordão, SP. Anais... Campos do Jordão: Sociedade Brasileira de Meteorologia, 1996b. 2v. 1250p. p.1113-1117.

HELDWEIN, A.B. Alerta fitossanitário. In: CONGRESSO BRASILEIRO DE AGROMETEOROLOGIA, 10., 1997, Piracicaba, SP. Suplemento dos Anais... Piracicaba: Sociedade Brasileira de Agrometeorologia, 1997. 82p. p.63-77.

MARENGO, J.A; OLIVEIRA, G.S. Impactos do fenômeno La Niña no tempo e clima do Brasil: desenvolvimento e intensificação do La Niña 1998/1999. In: CONGRESSO BRASILEIRO DE METEOROLOGIA, 10., 1998. Brasília, DF. Anais... Brasília: Sociedade Brasileira de Meteorologia, 1998. CD-ROM.

MATZENAUER, R. et al. Evapotranspiração da cultura do milho. II - Relações com a evaporação do tanque Classe A, com a evapotranspiração de referência e com a radiação solar global, em três épocas de semeadura. Revista Brasileira de Agrometeorologia, v.6, n.1, p.15-21, 1998. 
NOAA. ENSO: Cold and warm episodes by season. Capturado em 20 de janeiro de 2007. On line. Disponível na Internet: http://www.cpc.noaa.gov/products/analysis_ monitoring/insostuff/ensoyears.shtml.

OLIVEIRA, G.S; SATYAMURTY, P. O El Nino de 1997/98: Evolução e impactos no Brasil. In: CONGRESSO BRASILEIRO DE METEOROLOGIA, 10., 1998, Brasília, DF. Anais... Brasília: Sociedade Brasileira de Meteorologia, 1998. CD-ROM.
SENTELHAS, P.C. et al. Estimating leaf wetness duration on a cotton crop from meteorological data. Revista Brasileira de Agrometeorologia, v.12, n.2, p.235-245, 2004.

SOARES, R.V. Prevenção e controle de incêndios florestais. Curitiba: FUPEF, 1984. 160p.

VILELA, D. Sistema de conservação de forragem: fenação. Coronel Pacheco, MG: EMBRAPA- CNPGL, 1994. 34p. (Boletim de Pesquisa, 07). 